\title{
Trajectory Controllability of Dynamical Systems with Non-instantaneous Impulses
}

\author{
Vishant Shah ${ }^{1}$, Jaita Sharma ${ }^{1}$, Prakashkumar H. Patel ${ }^{2 *}$ \\ ${ }^{1}$ Department of Applied Mathematics, The M. S. University of Baroda, Vadodara, \\ India-390001 \\ 2 Department of Mathematics, Krishna School of Science, Drs. Kiran \& Pallavi Patel Global \\ University, Varnama, Vadodara, India-391243 \\ Email: vishantmsu83@gmail.com, jaita.sharma-appmath@msubaroda.ac.in, \\ prakash5881@gmail.com
}

\begin{abstract}
This manuscript considered the system governed by the non-instantaneous impulsive evolution control system and discusses trajectory controllability of the governed system with classical and nonlocal initial conditions over the general Banach space. The results of the trajectory controllability for governed systems are obtained through the concept of operator semigroup and Gronwall's inequality. This manuscript is also equipped with examples to illustrate the applications of derived results.
\end{abstract}

Keywords- Evolution Equation, Non-instantaneous impulses, Trajectory Controllability, Classical conditions, Non-local conditions

AMS Subject Classification- 4K35, 34K45, 93B05, 93C25.

\section{Introduction}

Impulsive differential equations play a very important role in studying the behavior of the phenomenon having abrupt changes in the physical problems. If the changes are at a fixed moment of time then it is called instantaneous impulsive differential equations and the changes are over the small intervals then it is called non-instantaneous impulsive differential equations. There is a wide range of applications of these impulsive evolution equations in all fields of sciences namely, physical sciences, biological science, environmental sciences. These applications are found in the monograph [1, 2, 3] and research articles [4, 5, 6, 7, 8] and references their in. Qualitative properties like existence and uniqueness of solution and continuity of the solutions of instantaneous and noninstantaneous differential or integrodifferential or evolution equations are found in research articles with initial conditions are found in research articles $[9,10,11,12,13,14,15,16,17,18,19,20,21,22,23,24,25,26,27,28$, and references therein.

Nowadays, the concept of controllability plays an important role in the field of applied mathematics. In the notion of controllability, one has to find the control which steers the initial state at the initial

${ }^{*}$ Corrorsponding Author 
time to desired final state at the final time. Complete controllability of finite-dimensional linear system using functional analytic approach was first introduced by Kalmann. Thereafter many researchers were involved in developing the different controllability of various linear and nonlinear finite and infinite dimensional impulsive and non-impulsive systems using the functional analytic approach. The notion of the controllability are found in the monographs [29, 30, 31] and articles [32, 33, 34, 35, 36, 37. and reference their in. Trajectory controllability is the strongest form of controllability amongst all other forms of controllability. The study of trajectory controllability of one-dimensional systems was initiated by George [39]. Thereafter Chalishajar, et. al. [40] generalized the concept of trajectory controllability on finite and infinite-dimensional systems.

This manuscript established the trajectory controllability of the system:

$$
\begin{array}{rrr}
x^{\prime}(\tau) & =A(\tau) x(\tau)+F(\tau, x(\tau))+W(\tau), & \tau \in\left[s_{k}, t_{k}+1\right), \text { for all } k=0,1,2 \cdots, p \\
x(\tau) & =G_{k}(\tau, x(\tau))+W_{k}(\tau), & \tau \in\left[t_{k}, s_{k}\right), \text { for all } k=1,2, \cdots, p,
\end{array}
$$

with local condition $x(0)=x_{0}$ and non-local condition $x(0)=x_{0}-h(x)$.

\section{Preliminaries}

This section discusses definitions and prepositions to establish trajectory controllability of the system governed by non-instantaneous impulsive evolution equation with classical as well nonlocal conditions.

Definition 2.1. The system (1.1) is completely controllable on the interval $\mathcal{J}=\left[0, T_{0}\right]$ if for any $x_{0}, x_{1} \in \mathcal{X}$, if there exist a control $W(\cdot) n$ in $\mathcal{U}$ (control space) steers the system from $x_{0}$ at $\tau=0$ to $x_{1}$ at $\tau=T_{0}$.

In the definition of complete controllability, there is no information of the path or trajectory on which the given system is to be driven. Sometimes this leads to high cost So to overcome this situation we select the path or trajectory (having minimum cost) under which the control system drives from $x_{0}$ to $x_{1}$ over the interval $\mathcal{J}$. Searching of controller $W(\cdot)$ in a way that the system drive from $x_{0}$ to $x_{1}$ over the interval is called trajectory controllability of the system. Therefore, trajectory controllability of the system is strongest amongst all types of controllability.

Definition 2.2. Let, $\mathcal{C}_{\mathcal{T}}$ be the set of all trajectories under which the system (1.1) drives from $x_{0}$ to $x_{1}$ over the interval $\mathcal{J}$. The system (1.1) is trajectory controllable if for any $z \in \mathcal{C}_{\mathcal{T}}$, there is a controller $W(\cdot) \in \mathcal{U}$ such that state of the system $x(\tau)$ drives on prescribed trajectory $z(\tau)$. This means $x(\tau)=z(\tau)$ a.e. over the interval $\mathcal{J}$.

\section{T-controllability with local conditions}

Consider the system governed by the non-instantaneous impulsive evolution equation

$$
\begin{aligned}
x^{\prime}(\tau) & =A(\tau) x(\tau)+F(\tau, x(\tau))+W(\tau), \quad \tau \in\left[s_{k}, t_{k+1}\right) \\
x(\tau) & =G_{k}(\tau, x(\tau))+W_{k}(\tau), \quad \tau \in\left[t_{k}, s_{k}\right) \\
x(0) & =x_{0}
\end{aligned}
$$

over the interval $\left[0, T_{0}\right]$. Here, $x(\tau)$ is the state of the system lies in Banach space $\mathcal{X}$ at any time $\tau \in\left[0, T_{0}\right], A(\tau)$ at any time $\tau$ is a linear operator on the Banach space $\mathcal{X}, F, G_{K}:\left[0, T_{0}\right] \times \mathcal{X} \rightarrow \mathcal{X}$ are nonlinear functions. 
To discuss trajectory controllability of the system governed by non-instantaneous impulsive evolution equation (3.1), we have following theorem:

\section{Theorem 3.1. If,}

(A1) Linear operator $A$ in the system (3.1) infinitesimal generator of $C_{0}$ semigroup.

(A2) The non-linear map $F:\left[0, T_{0}\right] \times \mathcal{X} \rightarrow \mathcal{X}$ is continuous such that there exist a non-decreasing function $l_{F}: \mathbb{R}_{+} \rightarrow \mathbb{R}+$ and positive real number $r_{0}$ satisfying

$$
\left\|F\left(\tau, x_{1}\right)-F\left(\tau, x_{2}\right)\right\| \leq l_{F}(r)\left\|x_{1}-x_{2}\right\|
$$

, for all $\tau \in\left[0, T_{0}\right], x_{1}, x_{2} \in B_{r}(\mathcal{X})$ and $r \leq r_{0}$.

(A3) The non-linear map $G_{k}:\left[0, T_{0}\right] \times \mathcal{X} \rightarrow \mathcal{X}$ for all $k$ are are continuous such that there exist constants $0<l_{g k}<1$ satisfying

$$
\left\|G_{k}\left(\tau, x_{1}\right)-G_{k}\left(\tau, x_{2}\right)\right\| \leq l_{g k}\left\|x_{1}-x_{2}\right\|
$$

, for all $\tau \in\left[0, T_{0}\right]$ and $l_{g}=\max \left\{l_{g k} ; \forall k\right\}$.

Then, the system (3.1) is trajectory controllable over the interval $\left[0, T_{0}\right]$.

Proof. Let $u(\tau)$ be any trajectory in $\mathcal{C}_{\tau}$ satisfying $x\left(t_{k}^{+}\right)=u\left(t_{k}^{+}\right)$along which the system (3.1) steered from initial state $x_{0}$ at $\tau=0$ to desired final state $x_{1}$ at $\tau=T_{0}$.

Over the interval $\left[0, t_{1}\right)$, the system (3.1) becomes:

$$
\begin{aligned}
x^{\prime}(\tau) & =A(\tau) x(\tau)+F(\tau, x(\tau))+W(\tau) \\
x(0) & =x_{0}
\end{aligned}
$$

Consider

$$
W(\tau)=u^{\prime}(\tau)-A(\tau)-F(\tau, u(\tau))
$$

over the interval $\left[0, t_{1}\right)$ in and plugging it in the 3.2 the system $(3.2)$ becomes

$$
x^{\prime}(\tau)=A(\tau) x(\tau)+F(\tau, x(\tau))+u^{\prime}(\tau)-A(\tau)-F(\tau, u(\tau))
$$

with initial condition $x(0)-u(0)=0$.

Choosing variable $z=x-u$ the equation system reduces to

$$
\begin{aligned}
z^{\prime}(\tau) & =A(\tau) z(\tau)+F(\tau, x(\tau))-F(\tau, u(\tau)) \\
z(0) & =0
\end{aligned}
$$

and problem of trajectory controllability of the system 3.2 is reduced to the solvability of the system (3.3) over the interval $\left[0, t_{1}\right)$. The mild solution of the system (3.3) is given by:

$$
z(\tau)=\int_{0}^{\tau} \mathcal{T}(\tau-\zeta)[F(\zeta, x(\zeta))-F(\zeta, u(\zeta))] d \zeta
$$


where, $\mathcal{T}(\tau)$ is $C_{0}$ semigroup generated by linear operator $A$ satisfying $\|\mathcal{T}(\tau)\| \leq M$ for some positive number $M$.

Therefore,

$$
\begin{aligned}
\|z(\tau)\| & \leq \int_{0}^{\tau}\|\mathcal{T}(\tau-\zeta)\|\|[F(\zeta, x(\zeta))-F(\zeta, u(\zeta))]\| d \zeta \\
& \leq M \int_{0}^{\tau} l_{F}(r)\|x(\zeta)-u(\zeta)\| d \zeta \\
& \leq M \int_{0}^{\tau} l_{F}(r)\|z(\zeta)\| d \zeta
\end{aligned}
$$

and using Gronwall's inequality, we obtain $z(\tau)=0$ over the interval $\left[0, t_{1}\right)$. Hence, $x(\tau)=u(\tau)$ for all $\tau \in\left[0, t_{1}\right)$. Therefore, the system is trajectory controllable over the interval $\left[0, t_{1}\right)$.

Over the interval $\left[t_{k}, s_{k}\right)$, the system becomes

$$
x(\tau)=G_{k}(\tau, x(\tau))+W_{k}(\tau),
$$

and at $\tau=t_{k}^{+}$value of the state $x$ is given by $x\left(t_{k}^{+}\right)=G_{k}\left(t_{k}^{+}, x\left(t_{k}^{+}\right)\right)+W_{k}\left(t_{k}^{+}\right)$.

Plugging the control $W_{k}(\tau)=u(\tau)-G_{k}(\tau, x(\tau))$ over the interval $\left[t_{k}, s_{k}\right)$ in the system (3.5) the system becomes:

$$
x(\tau)-u(\tau)=G_{k}(\tau, x(\tau))-G_{k}(\tau, u(\tau))
$$

Choosing $z(\tau)=x(\tau)-u(\tau)$ we obtain

$$
z(\tau)=G_{k}(\tau, x(\tau))-G_{k}(\tau, u(\tau))
$$

and the value of the $z$ at $\tau=t_{k}^{+}$is zero. Therefore, we have

$$
\|z(\tau)\| \leq\left\|G_{k}(\tau, x(\tau))-G_{k}(\tau, u(\tau))\right\| \leq l_{g}\|z(\tau)\|,
$$

using (A3) $l_{g}<1$ we obtain $z(\tau)=0$ for all $\tau \in\left[t_{k}, s_{k}\right.$ ) Therefore, system (3.1) is T-Controllable over the interval $\left[t_{k}, s_{k}\right)$. Moreover, $z\left(s_{k}\right)=0$ as $G_{K}^{\prime} s$ are continuous.

Over the interval $\left[s_{k}, t_{k+1}\right)$ the system (3.1) becomes:

$$
\begin{aligned}
& x^{\prime}(\tau)=A(\tau) x(\tau)+F(\tau, x(\tau))+W(\tau) \\
& x\left(s_{k}\right)=u\left(s_{k}\right)
\end{aligned}
$$

Choose the control over the interval $\left[s_{k}, t_{k+1}\right.$ as:

$$
W(\tau)=u^{\prime}(\tau)-A(\tau)-F(\tau, u(\tau))
$$

and plugging it in the equation 3.6 we get,

$$
x^{\prime}(\tau)=A(\tau) x(\tau)+F(\tau, x(\tau))+u^{\prime}(\tau)-A(\tau)-F(\tau, u(\tau))
$$

considering $z(\tau)=x(\tau)-u(\tau)$, above expression becomes:

$$
\begin{aligned}
& z^{\prime}(\tau)=A(\tau) z(\tau)+F(\tau, x(\tau))-F(\tau, u(\tau)) \\
& z\left(s_{k}\right)=0
\end{aligned}
$$


Therefore

$$
\begin{aligned}
\|z(\tau)\| & \leq \int_{s_{k}}^{\tau}\|\mathcal{T}(\tau-\zeta)\|\|[F(\zeta, x(\zeta))-F(\zeta, u(\zeta))]\| d \zeta \\
& \leq M \int_{0}^{\tau} l_{F}(r)\|x(\zeta)-u(\zeta)\| d \zeta \\
& \leq M \int_{0}^{\tau} l_{F}(r)\|z(\zeta)\| d \zeta
\end{aligned}
$$

and using Gronwall's inequality, we obtain $z(\tau)=0$ over the interval $\left[s_{k}, t_{k+1}\right)$. Hence, $x(\tau)=u(\tau)$ for all $\tau \in\left[s_{k}, t_{k+1}\right)$. Therefore, the system is $\mathrm{T}$ - controllable over the interval $\left[s_{k}, t_{k+1}\right)$.

Since, the system is T-controllable over the intervals $\left[0, t_{1}\right),\left[s_{k}, t_{k+1}\right)$ and $\left[t_{k}, s_{k}\right)$ for all $k$. Hence, the system is controllable over entire interval $\left[0, T_{0}\right]$. This completes the proof of the theorem.

Example 3.1. Let, $\mathcal{X}=L^{2}([0, \pi], \mathbb{R})$ and consider the system governed by non-instantaneous impulsive evolution equation:

$$
\begin{array}{rlr}
\frac{\partial H(\tau, \psi)}{\partial \tau}=\partial_{\psi}^{2} H(\tau, \psi)+F(\tau, H(\tau, \psi))+w(\tau, \psi) & \tau \in[0,1 / 3) \cup[2 / 3,1], \\
H(\tau, \psi)=G_{1}(\tau, H(\tau, \psi))+w_{1}(\tau, \psi) & \tau \in[1 / 3,2 / 3), \\
H(\tau, 0)=0 \quad H(\tau, \pi)=0 & \tau>0, \\
H(0, \psi)=H_{0}(\psi) & 0<\psi<\pi,
\end{array}
$$

over the interval $[0,1]$.

Defining the operator on the space $\mathcal{X}$ as $A(\tau)=\partial_{\psi}^{2}, A(\tau)$ is the infinitesimal generator of the $C_{0}$ semigroup $\mathcal{T}(\tau)$. The representation of $\mathcal{T}(\tau)$ is

$$
\mathcal{T}(\tau) z=\sum_{m=0}^{\infty} \exp \left(\mu_{m} \tau\right)<z, \phi_{m}>\phi_{m}
$$

where, $\phi_{m}=\sqrt{2} \sin (n \psi)$ for all $m=1,2, \cdots$ is the orthonormal basis corresponding to eigenvalue $\mu_{m}=-m^{2}$ of the operator $A$.

With this concept the equation (3.8) can be rewritten as and abstract equation on the space $\mathcal{X}$ as

$$
\begin{aligned}
x^{\prime}(\tau) & =A(\tau)+F(\tau, x)+W(\tau) & \tau \in[0,1 / 3) \cup[2 / 3,1], \\
x(\tau) & =G_{1}(\tau, x) & \tau \in[1 / 3,2 / 3), \\
x(0) & =x_{0}, &
\end{aligned}
$$

where, $x(\tau)=H(\tau, \cdot), W(\tau)=w(\tau, \cdot)$ and $W_{1}(\tau)=w_{1}(\tau, \psi)$. The system (3.9) is trajectory controllable over the interval $[0,1]$ if the functions $F$ and $G_{1}$ satisfies the hypotheses of the theorem.

\section{T-controllability with non-local conditions}

Consider the system governed by the non-instantaneous impulsive evolution equation

$$
\begin{aligned}
x^{\prime}(\tau) & =A(\tau) x(\tau)+F(\tau, x(\tau))+W(\tau), \quad \tau \in\left[s_{k}, t_{k+1}\right) \\
x(\tau) & =G_{k}(\tau, x(\tau))+W_{k}(\tau), \quad \tau \in\left[t_{k}, s_{k}\right) \\
x(0) & =h(x)
\end{aligned}
$$


over the interval $\left[0, T_{0}\right]$. Here, $x(\tau)$ is the state of the system lies in Banach space $\mathcal{X}$ at any time $\tau \in\left[0, T_{0}\right], A(\tau)$ at any time $\tau$ is a linear operator on the Banach space $\mathcal{X}, F, G_{K}:\left[0, T_{0}\right] \times \mathcal{X} \rightarrow \mathcal{X}$ are nonlinear functions and $h: \mathcal{X} \rightarrow \mathcal{X}$ is the operator representing the non-local conditions. The mild solution of the equation 4.1 is given by

$$
x(\tau)=\left\{\begin{array}{lr}
\mathcal{T}(\tau) h(x)+\int_{0}^{\tau} \mathcal{T}(\tau-\zeta) F(\zeta, x(\zeta)) d \zeta, & \tau \in\left[0, t_{1}\right) \\
G_{k}(\tau, x(\tau)), & \tau \in\left[t_{k}, s_{k}\right) \\
\mathcal{T}(\tau) G_{k}\left(s_{k}, x\left(s_{k}\right)\right)+\int_{s_{k}}^{\tau} \mathcal{T}(\tau-\zeta) F(\zeta, x(\zeta)) d \zeta, & \tau \in\left[s_{k}, t_{k+1}\right),
\end{array}\right.
$$

where, $\mathcal{T}(\tau)$ is semigroup generated by the linear operator $A(\tau)$.

The following theorem discusses the trajectory controllability of the system governed by the equation 4.1.

Theorem 4.1. If,

(A1) Linear operator $A$ in the system (3.1) infinitesimal generator of $C_{0}$ semigroup.

(A2) The non-linear map $F:\left[0, T_{0}\right] \times \mathcal{X} \rightarrow \mathcal{X}$ is continuous such that there exist a non-decreasing function $l_{F}: \mathbb{R}_{+} \rightarrow \mathbb{R}+$ and positive real number $r_{0}$ satisfying

$$
\left\|F\left(\tau, x_{1}\right)-F\left(\tau, x_{2}\right)\right\| \leq l_{F}(r)\left\|x_{1}-x_{2}\right\|
$$

, for all $\tau \in\left[0, T_{0}\right], x_{1}, x_{2} \in B_{r}(\mathcal{X})$ and $r \leq r_{0}$.

(A3) The non-linear map $G_{k}:\left[0, T_{0}\right] \times \mathcal{X} \rightarrow \mathcal{X}$ for all $k$ are are continuous such that there exist constants $0<l_{g k}<1$ satisfying

$$
\left\|G_{k}\left(\tau, x_{1}\right)-G_{k}\left(\tau, x_{2}\right)\right\| \leq l_{g k}\left\|x_{1}-x_{2}\right\|
$$

for all $\tau \in\left[0, T_{0}\right]$ and $l_{g}=\max \left\{l_{g k} ; \forall k\right\}$.

(A4) The function $h: \mathcal{X} \rightarrow \mathcal{X}$ is Lipchitz continuous with Lipchitz constant $0 \leq l_{h} \leq 1$.

Then, the system 4.1) is trajectory controllable over the interval $\left[0, T_{0}\right]$.

Proof. Let $u(\tau)$ be any trajectory in $\mathcal{C}_{\tau}$ satisfying $x\left(t_{k}^{+}\right)=u\left(t_{k}^{+}\right)$along which the system 4.1) steered from initial state $x(0)=h(x)$ at $\tau=0$ to desired final state $x_{1}$ at $\tau=T_{0}$.

Over the interval $\left[0, t_{1}\right)$, the system (4.1) becomes:

$$
\begin{aligned}
x^{\prime}(\tau) & =A(\tau) x(\tau)+F(\tau, x(\tau))+W(\tau) \\
x(0) & =h(x)
\end{aligned}
$$

Consider

$$
W(\tau)=u^{\prime}(\tau)-A(\tau)-F(\tau, u(\tau))
$$

over the interval $\left[0, t_{1}\right)$ and plugging it in the system (4.3), the system becomes

$$
x^{\prime}(\tau)=A(\tau) x(\tau)+F(\tau, x(\tau))+u^{\prime}(\tau)-A(\tau)-F(\tau, u(\tau))
$$


with initial condition $x(0)-u(0)=h(x)-h(u)$.

Choosing variable $z=x-u$ the equation system reduces to

$$
\begin{aligned}
z^{\prime}(\tau) & =A(\tau) z(\tau)+F(\tau, x(\tau))-F(\tau, u(\tau)) \\
z(0) & =h(x)-h(u)
\end{aligned}
$$

and problem of trajectory controllability of the system 4.3 is reduced to the solvability of the system (4.4) over the interval $\left[0, t_{1}\right)$. The mild solution of the system 4.4 is given by:

$$
z(\tau)=\mathcal{T}(\tau)[h(x)-h(u)]+\int_{0}^{\tau} \mathcal{T}(\tau-\zeta)[F(\zeta, x(\zeta))-F(\zeta, u(\zeta))] d \zeta
$$

where, $\mathcal{T}(\tau)$ is $C_{0}$ semigroup generated by linear operator $A$ satisfying $\|\mathcal{T}(\tau)\| \leq M$ for some positive number $M$.

Therefore,

$$
\begin{aligned}
\|z(\tau)\| & \leq\|\mathcal{T}(\tau)\|\|h(x)-h(u)\|+\int_{0}^{\tau}\|\mathcal{T}(\tau-\zeta)\|\|[F(\zeta, x(\zeta))-F(\zeta, u(\zeta))]\| d \zeta \\
& \leq M l_{h}\|x(\tau)-u(\tau)\|+M \int_{0}^{\tau} l_{F}(r)\|x(\zeta)-u(\zeta)\| d \zeta \\
& \leq M l_{h}\|z(\tau)\|+M \int_{0}^{\tau} l_{F}(r)\|z(\zeta)\| d \zeta
\end{aligned}
$$

This implies

$$
\|z(\tau)\| \leq \frac{M l_{F}(r)}{1-M l_{h}} \int_{0}^{\tau}\|z(\zeta)\| d \zeta
$$

Using Gronwall's inequality, we obtain $z(\tau)=0$ over the interval $\left[0, t_{1}\right)$. Hence, $x(\tau)=u(\tau)$ for all $\tau \in\left[0, t_{1}\right)$. Therefore, the system (4.1) is trajectory controllable over the interval $\left[0, t_{1}\right)$.

Over the interval $\left[t_{k}, s_{k}\right)$, the system becomes

$$
x(\tau)=G_{k}(\tau, x(\tau))+W_{k}(\tau),
$$

and at $\tau=t_{k}^{+}$value of the state $x$ is given by $x\left(t_{k}^{+}\right)=G_{k}\left(t_{k}^{+}, x\left(t_{k}^{+}\right)\right)+W_{k}\left(t_{k}^{+}\right)$.

Plugging the control $W_{k}(\tau)=u(\tau)-G_{k}(\tau, x(\tau))$ over the interval $\left[t_{k}, s_{k}\right)$ in the system (4.6) the system becomes:

$$
x(\tau)-u(\tau)=G_{k}(\tau, x(\tau))-G_{k}(\tau, u(\tau))
$$

Choosing $z(\tau)=x(\tau)-u(\tau)$ we obtain

$$
z(\tau)=G_{k}(\tau, x(\tau))-G_{k}(\tau, u(\tau))
$$

and the value of the $z$ at $\tau=t_{k}^{+}$is zero. Therefore, we have

$$
\|z(\tau)\| \leq\left\|G_{k}(\tau, x(\tau))-G_{k}(\tau, u(\tau))\right\| \leq l_{g}\|z(\tau)\|,
$$

using (A3) $l_{g}<1$ we obtain $z(\tau)=0$ for all $\tau \in\left[t_{k}, s_{k}\right)$ and using continuity of $G_{k}$ leads to $z\left(s_{k}\right)=0$. Therefore, system (4.6) is T-Controllable over the interval $\left[t_{k}, s_{k}\right)$.

Over the interval $\left[s_{k}, t_{k+1}\right)$ the system (3.1) becomes:

$$
\begin{aligned}
& x^{\prime}(\tau)=A(\tau) x(\tau)+F(\tau, x(\tau))+W(\tau) \\
& x\left(s_{k}\right)=u\left(s_{k}\right)
\end{aligned}
$$


Choose the control over the interval $\left[s_{k}, t_{k+1}\right.$ as:

$$
W(\tau)=u^{\prime}(\tau)-A(\tau)-F(\tau, u(\tau))
$$

and plugging it in the equation 4.7) we get,

$$
x^{\prime}(\tau)=A(\tau) x(\tau)+F(\tau, x(\tau))+u^{\prime}(\tau)-A(\tau)-F(\tau, u(\tau))
$$

, considering $z(\tau)=x(\tau)-u(\tau)$, above expression becomes:

$$
\begin{aligned}
& z^{\prime}(\tau)=A(\tau) z(\tau)+F(\tau, x(\tau))-F(\tau, u(\tau)) \\
& z\left(s_{k}\right)=0
\end{aligned}
$$

Therefore,

$$
\begin{aligned}
\|z(\tau)\| & \leq \int_{s_{k}}^{\tau}\|\mathcal{T}(\tau-\zeta)\|\|[F(\zeta, x(\zeta))-F(\zeta, u(\zeta))]\| d \zeta \\
& \leq M \int_{0}^{\tau} l_{F}(r)\|x(\zeta)-u(\zeta)\| d \zeta \\
& \leq M \int_{0}^{\tau} l_{F}(r)\|z(\zeta)\| d \zeta
\end{aligned}
$$

and using Gronwall's inequality, we obtain $z(\tau)=0$ over the interval $\left[s_{k}, t_{k+1}\right)$. Therefore, $x(\tau)=u(\tau)$ for all $\tau \in\left[s_{k}, t_{k+1}\right)$. Thus, the system is trajectory controllable over the interval $\left[s_{k}, t_{k+1}\right)$.

Since, the system is T-controllable over the intervals $\left[0, t_{1}\right),\left[s_{k}, t_{k+1}\right)$ and $\left[t_{k}, s_{k}\right)$ for all $k$. Hence, the system is controllable over entire interval $\left[0, T_{0}\right]$. This completes the proof of the theorem.

Example 4.1. Let, $\mathcal{X}=L^{2}([0, \pi], \mathbb{R})$ and consider the system governed by non-instantaneous impulsive evolution equation:

$$
\begin{aligned}
\frac{\partial H(\tau, \psi)}{\partial \tau}=\partial_{\psi}^{2} H(\tau, \psi)+F(\tau, H(\tau, \psi))+w(\tau, \psi) & \tau \in[0,1 / 3) \cup[2 / 3,1], \\
H(\tau, \psi)=G_{1}(\tau, H(\tau, \psi))+w_{1}(\tau, \psi) & \tau \in[1 / 3,2 / 3), \\
H(\tau, 0)=0 \quad H(\tau, \pi)=0 & \tau>0, \\
H(0, \psi)=H(\tau, \psi) & 0<\psi<\pi,
\end{aligned}
$$

over the interval $[0,1]$. Here, $H(\tau, \psi)$ is nonlocal opertor defined by $\sum_{i=1}^{n} \alpha_{i} H\left(\tau_{i}, \psi\right)$.

Defining the operator on the space $\mathcal{X}$ as $A(\tau)=\partial_{\psi}^{2}, A(\tau)$ is the infinitesimal generator of the $C_{0}$ semigroup $\mathcal{T}(\tau)$. The representation of $\mathcal{T}(\tau)$ is

$$
\mathcal{T}(\tau) z=\sum_{m=0}^{\infty} \exp \left(\mu_{m} \tau\right)<z, \phi_{m}>\phi_{m}
$$

where, $\phi_{m}=\sqrt{2} \sin (n \psi)$ for all $m=1,2, \cdots$ is the orthonormal basis corresponding to eigenvalue $\mu_{m}=-m^{2}$ of the operator $A$.

With this concept the equation (4.9) can be rewritten as and abstract equation on the space $\mathcal{X}$ as

$$
\begin{aligned}
x^{\prime}(\tau) & =A(\tau)+F(\tau, x)+W(\tau) & \tau \in[0,1 / 3) \cup[2 / 3,1], \\
x(\tau) & =G_{1}(\tau, x) & \tau \in[1 / 3,2 / 3), \\
x(0) & =h(x), &
\end{aligned}
$$

where, $x(\tau)=H(\tau, \cdot), W(\tau)=w(\tau, \cdot), W_{1}(\tau)=w_{1}(\tau, \psi)$ and $h(x)=\sum_{i=1}^{n} \alpha_{i} x\left(\tau_{i}\right)$. The system (4.10) is trajectory controllable over the interval $[0,1]$ if the functions $F, G_{1}$ and $h$ satisfies the hypotheses of the theorem. 


\section{Conclusion}

In this manuscript, we have discussed the trajectory controllability of the system governed by noninstantaneous impulsive evolution equation with classical as well as nonlocal conditions on the infinite dimensional Banach space.

\section{References}

[1] V. Lakshmikantham, D. D. Bainov and P. S. Simeonov, Theory of Impulsive Differential Equations, World Scientific, Singapore, 1989.

[2] A. M. Samoilenko, N. A Perestyuk, Impulsive Differential Equations, World Scientific, Singapore, 1995.

[3] R. Agarwal, S. Hristova, D. O'Regan, Non-Instantaneous Impulses in Differential Equations, Springer International Publishing, Switzerland, 2017

[4] D. D. Bainov and A. B. Dishliev, Population dynamics control in regard to minimizing the time necessary for the regeneration of a biomass taken away from the population, Appl.Math.Comput. 39 (1990), 37-48.

[5] L. H. Erbe, H. I. Freedman, X. Z. Liu and J. H. Wu, Comparison principles for impulsive parabolic equations with applications to models of single species growth, J. Austral. Math. Soc. 32(1991), $382-400$.

[6] M. Kirane and Yu. V. Rogovchenko, Comparison results for systems of impulse parabolic equations with applications to population dynamics, Nonlinear Anal. 28 (1997), 263-277.

[7] E. Joelianto, Linear impulsive differential equations for hybrid system modelling, in: 2003 European Control Conference(ECC), pp. 3335-3340, Cambridge, UK, 1-4 Sept. 2003.

[8] K. Dishlieva, Impulsive differential equations and applications, J. Appl. Comput. Math. 2016 (2016), 1-6.

[9] V. Milman and A. Myshkis, On the stability of motion in the presence of impulses, Siberian Math. J. 1 (1996), 233-237.

[10] Y. V. Rogovchenko, Nonlinear impulse evolution systems and applications to population models, J. Math. Anal. App. 207 (1997), 300-315.

[11] Y. Rogovchenko, Impulsive evolution systems: main results and new trends, Dynamics Contin. Diser. Impulsive Sys. 3(1997), 57-88.

[12] J. H. Liu, Nonlinear impulsive evolution equations, Dynamics Contin. Diser. Impulsive System 6 (1999), 77-85.

[13] H. Yang, Positive solutions for the initial value problems of impulsive evolution equations, J. Math. Res. Exposition 31(2011), 1047-1056.

[14] X. Xiang, Y. Peng and W. Wei, A general class of nonlinear impulsive integral differential equations and optimal control on Banach space, Discrete Cont. Dynemical Syst. 2005 (2005), 911-919. 
[15] A. Anguraj and M. M. Arjunan, Existence and uniqueness of mild and classical solutions of impulsive evolution equations, Ele. J. Diff. Eq. 111 (2005), 1-8.

[16] X. Zhang, Solutions of semilinear evolution equations of mixed type in Banach spaces, Acta Anal. Funct. Appl. 11 (2009), 363-368.

[17] P. Sattayatham, Strongly nonlinear impulsive evolution equations and optimal control, Nonlinear Anal. 57 (2004), 1005-1020.

[18] J. Liang, J. H. Liu and T.-J. Xiao, Nonlocal impulsive problems for nonlinear differential equations in Banach spaces, Math.v Comput. Modell. 49 (2009), 798-804.

[19] Z. Fan and G. Li, Existence results for semilinear differential equations with nonlocal and impulsive conditions, J. Funct. Anal. 258 (2010), 1709-1727.

[20] S. Wen and S. Ji, An existence theorem of impulsive differential equations with nonlocal conditions, Int. J. Nonlinear Sci. 14 (2012), 186-192.

[21] Q. Tang and J. J. Nieto, Variational approach to impulsive evolution equations, Appl. Math. Lett. 36 (2014), 31-35.

[22] P. Chen and J. Mu, Monotone iterative method for semilinear impulsive evolution equation of mixed type in Banach space, Electron. J. Differ. Eq. 2010 (2010), 1-13.

[23] P. Chen and Y. Li, Mixed monotone iterative technique for a class of semilinear impulsive evolution equations in Banach spaces, Nonlinear Anal. 74 (2011), 3578-3588.

[24] P. Chen, Y. Li and H. Yang, Perturbation method for nonlocal impulsive evolution equations, Nonlinear Anal. Hybrid Syst. 8(2013), 22-30.

[25] T. Cardinali and P. Rubbioni, Mild solutions for impulsive semilinear evolution differential equation, J. Appl. Funct. Anal. 1 (2006), 303-325.

[26] V. Shah, J. George, J. Sharama and P. Muthukumar, Existence and Uniqueness of Classical and Mild Solutions of Generalized Impulsive Evolution Equation, Int. J. Nonlinear Sci. Numer. Simul. 19 (2018), 775-780.

[27] E. Hernandez, M. Pierri, D. O'Regan, On Abstract Differential Equations with Non instantaneous impulses, Topological Methods in Nonlinear Analysis, 46(2015), 1067-1088.

[28] A. Sood, S. Srivastava, On Stability of Differential Systems with Noninstantaneous Impulses, Mathematical Problems in Engineering, 2015 (2015), 1-5.

[29] D. L. Russell, Mathematics of Finite-dimensional Control Systems: Theory and Design, M. Dekker, New York, 1979.

[30] E. D. Sontag, Deterministic Finite Dimensional Systems, Springer, 1998.

[31] R. W. Brockett, Finite Dimensional Linear Systems, John Wiley \& Sons, USA 1970.

[32] M. C. Joshi, R. K. George, Controllability of Nonlinear Systems, Numerical Functional Analysis \& Optimization, 10(1989), 139-166. 
[33] J. Klamka, Schauder's fixed-point theorm in Nonlinear Controllability problems, Control and Cybernetics, 29(2000), 153-165.

[34] Z. G. Li, Y. Wen and Y. C. Soh, Analysis and design of impulsive control, IEEE Trans. Autom. Control 46(2001), 894-897.

[35] R. K. George, Approximate controllability of non-autonomous semilinear systems, Nonlinear Anal., 24 (1995), 1377-1393.

[36] J. Klamka, A. Babiarz and M. Niezabitowski, Banach fixed-point theorem in semilinear controllability problems-a survey, Bull. Polish Acad. Sci. Tech. Sci., 64 (2016), 21-35.

[37] J. Klamka, A. Babiarz and M. Niezabitowski, Schauder's fixed point theorem in approximate controllability problems, Int. J. Appl. Math. Comput. Sci., 26 (2016), 263-275.

[38] P. Chen, X. Zhang and Y. Li, Approximate controllability of non-autonomous evolution system with nonlocal conditions, J. Dyn. Control Syst., 26 (2020), 1-16.

[39] R. K. George, Trajectory controllability of 1-dimensional nonlinear systems, Proceedings of the Research Seminar in honour of Professor M.N. Vasavada, Anand, India: S.P. University 1996 (pp. 43-48).

[40] D. N. Chalishajar, R. K. George, A. K. Nandkumaran, F. S. Acharya, Trajectory controllability of nonlinear integro-differential system, Journal of The Franklin Institute, 347(2010), 1065-1075. 